\title{
Características morfológicas de aneurimas cerebrales del polígono de Willis: estudio anatómico directo
}

\author{
S.T. Quintero-Oliveros*; L.E. Ballesteros-Acuña*; J.O. Ayala-Pimentel* y P.L. Forero-Porras*,** \\ *Departamento de Ciencias Basicas. Universidad Industrial de Santander. **Instituto de Medicina Legal y Ciencias Forenses. Bucaramanga. \\ Colombia.
}

Resumen

Objetivo. Determinar la prevalencia y las características morfológicas de los aneurismas sin ruptura del círculo arterial del cerebro en una muestra de población mestiza colombiana.

Materiales y métodos. Mediante abordaje y canalización de las arterias carótidas comunes y vertebrales se realizó inyección de resina mezclada con rojo mineral a 125 encéfalos de individuos masculinos, entre 16 y 65 años, raza mestiza, a quienes se les practicó autopsia en el Instituto de Medicina Legal, Bucaramanga - Colombia. Se realizó exéresis y fijación de los encéfalos, posteriormente bajo lupa de aumento se identificaron los segmentos del círculo arterial del cerebro y las lesiones aneurismáticas ubicadas a este nivel.

Resultados. Se observaron nueve aneurismas en seis cerebros $(\mathbf{4 . 8 \%})$; la localización mas frecuente fue el segmento supraclinoideo de la arteria carótida interna, con 4 casos $(44.4 \%)$ de los cuales 3 se ubicaron en el origen de la arteria coroidea anterior. En la arteria comunicante anterior se localizaron 3 aneurismas (33.3\%); los casos restantes se ubicaron, uno (11.1\%) en el segmento precomunicante de la arteria cerebral anterior y otro en la bifurcación de la arteria basilar. La media del tamaño de las lesiones aneurismáticas fue de $2.49 \mathrm{~mm}$ DS 0.37. Los encéfalos con aneurismas presentaron concomitantemente alguna variación en la configuración del círculo arterial del cerebro, con predominio de la hipoplasia de la arteria comunicante posterior.

Conclusiones. La prevalencia de aneurismas en la muestra evaluada es similar a lo informado en otros grupos poblacionales. Se destaca la presencia de aneurismas en el origen de la arteria coroidea anterior, localización señalada en la literatura como poco frecuente.

PALABRAS CLAVE: Anatomía vascular. Aneurismas cerebrales. Polígono de Willis. Raza mestiza.

Recibido: 24-06-08. Aceptado: 25-11-08
Morphological characteristics of cerebral aneurysm of Willis' circle: a direct anatomical study

Summary

Objective. To determine the prevalence and morphologic characteristics of unruptured aneurysms of Willis' circle in a sample of mestizo Colombian population.

Materials and methods. A mixture of resin and mineral red was injected into cerebral arteries by dissection and canalization of common carotids and vertebral arteries of the encephalons of $\mathbf{1 2 5}$ mestizo male cadavers of 16 to 65 years old. The procedure was carried out during the autopsy course at the Legal Medicine Institute, Bucaramanga - Colombia. Then the encephalons were extracted and fixed. After that, the Willis' circles were identified and the presence of aneurysms at this level was determined with magnifying glass.

Results. A total of nine aneurysms were observed in six brains $(4.8 \%)$. The most frequent location was the supraclinoid segment of the intern carotid artery, with 4 cases $(44.4 \%)$, three of which were localized in the origin of the anterior choroidal artery. Additionally, three aneurysms were found in the anterior communicating artery $(33.3 \%)$. From the remaining cases, one $(\mathbf{1 1 . 1 \% )}$ was localized in the pre-communicating segment of the anterior cerebral artery, and the other in the bifurcation of the basilar artery. The average size of the aneurysms was $2.49 \mathrm{~mm}$ SD 0.37 . The affected encephalons presented concomitant variations of the Willis' circle configuration, being predominant the presence of hypoplasic posterior communicanting arteries.

Conclusion. The aneurysm prevalence in the evaluated sample was similar to the reported in other populations. In this work, the presence of aneurysms on the origin of the anterior chorioid artery, an unusually

Abreviaturas. (ACA): arteria cerebral anterior. (ACI): arteria carótida interna. (ACP) arteria cerebral posterior. (AcoA): arteria comunicante anterior. (AcoP): arteria comunicante posterior. $(P W)$ : polígono de Willis. 
reported localization, was prominent.

KEY WORDS: Vascular anatomy. Cerebral aneurysm. Circle of Willis. Mestizo population

\section{Introducción}

Los aneurismas intracraneales son considerados la principal anormalidad cerebrovascular corregible quirúrgicamente y son una causa importante de hemorragia subarac-noidea en la población adulta ${ }^{4}$ Esta patología ha sido descrita con una prevalencia del $0.2-9.9 \%$ en la población general, con una tasa de ruptura anual de1-2\% $\%^{1,3,4,13,15,19,24}$.

La prevalencia e incidencia de los aneurismas cerebrales en la población colombiana no es bien conocida, dado que los estudios epidemiológicos se han limitado a establecer el evento agudo de la enfermedad cerebrovascular sin determinar su etiología. Los estudios colombianos reportan una prevalencia de enfermedad cerebrovascular de 3.1/1000 habitantes, siendo mayor en edades superiores a 50 años donde alcanza una prevalencia de 19.9/1000 habitantes. La enfermedad cerebrovascular representa la cuarta causa de mortalidad en Colombia y el $6.7 \%$ del total de muertes del $2005^{27,29,30}$. Se ha reportado que el 5\% de la enfermedad cerebrovascular corresponde a hemorragia subaracnoidea; a su vez la mayoría de los casos ocurren a consecuencia de la ruptura de un aneurisma con hemorragias fatales en un $50 \%{ }^{5,6,26}$. Estos datos dan relevancia a esta entidad y permiten centrar la discusión en el costobeneficio de implementar programas de detección precoz, evaluación y seguimiento prolongado que logre disminuir el impacto y los costos en salud derivados del desenlace de estas lesiones ${ }^{2,6,25}$.

La mayoría de los aneurismas cerebrales, se localizan en los segmentos del polígono de Willis y los cuatro primeros milímetros de la arteria cerebral media. Los aneurismas localizados en la arteria carótida interna y en el segmento posterior del polígono tienen mayor riesgo de ruptura que aquéllos localizados en el segmento anterior o en la arteria cerebral media. Adicionalmente se ha señalado que en el $70 \%$ de los casos los aneurismas intracraneales tienen un tamaño inferior o igual a $10 \mathrm{~mm}$; por encima de este tamaño se ha determinado un alto riesgo de crecimiento y ruptura $3,8,13,19,24 ;$ sin embargo, este evento ha sido reportado en un rango $82-85.6 \%$ de los casos en aneurismas menores a $10 \mathrm{~mm}^{2,6,8,25}$.

Se ha postulado que las variaciones morfológicas de los vasos del círculo de Willis son un factor de riesgo involucrado en la progresión y ruptura de las lesiones aneurismáticas, por lo cual múltiples estudios quirúrgicos, imagenológicos, y de autopsia en diferentes grupos raciales y étnicos han establecido su asociación ${ }^{1-3,6,8,14,16,18,25}$.

Al no contar con información en población mestiza colombiana, este estudio plantea determinar la prevalencia y expresión morfológica de los aneurismas sin ruptura del polígono de willis en una muestra de este grupo poblacional.

\section{Materiales y métodos}

En este estudio morfológico no probabilístico y descriptivo transversal se evaluaron los Polígonos de Willis obtenidos de 125 cadáveres frescos fallecidos violentamente, a quienes se les practicó necropsia en el Instituto de Medicina Legal y Ciencias Forenses de Bucaramanga, Colombia. Esta investigación fue realizada de acuerdo a las normas colombianas vigentes (decreto 008430 de 1993) y fue catalogada como una investigación con riesgo mínimo. Se obtuvo una muestra por conveniencia, que cumpliera con los siguientes criterios de inclusión: encéfalos de sujetos masculinos, con edades entre 16 y 65 años; del grupo racial mestizo (se consideraron mestizos a los individuos que presentaron mezcla fenotípica de los grupos raciales caucasoide, negroide y mongoloide), sin evidencia de haber fallecido por traumatismo directo o patologías que comprometieran al sistema nervioso central.

Cada espécimen fue sometido a cateterización bilateral de las arterias carótidas internas y vertebrales, a través de éstas se practicó lavado y prefijado del lecho vascular cerebral con formaldehído al $10 \%$ y posterior inyección de $100 \mathrm{cc}$ de resina semisintética (mezcla de palatal E210® basf, colombia, estireno al $9.8 \%$ y $0.2 \%$ de octoacto de cobalto al 6\%) impregnada de color rojo mineral. Una vez obtenida la polimerización de la resina (30 minutos), se practicó la exéresis del encéfalo. Posteriormente la muestra se sometió a fijación con formaldehído al 10\% durante 10 días. Esta metodología de fijación de los lechos vasculares posterior a la polimerización de la resina, nos permite no considerar el factor de corrección del tamaño de las lesiones aneurismáticas propuesto por algunos autores ${ }^{13,20,23}$ debido a que la resina polimerizada impide la disminución de las dimensiones de los vasos al someter los encéfalos a fijación. Los lechos vasculares fueron disecados utilizando lupa de 4X. Se realizó la medición de la longitud y calibre del polígono de Willis (PW) considerando los siguientes segmentos: arteria carótida interna (ACI) desde su segmento supraclinoideo hasta la emergencia de la cerebral anterior; arterias cerebrales anterior (ACA) y posterior (ACP) desde el punto de origen hasta el punto de unión con las arterias comunicantes y las arterias comunicantes anterior (AcoA) y posterior $(\mathrm{AcoP})^{16}$; se realizó la medición del tamaño de los aneurismas determinado como el promedio de tres dimensiones (altura, espesor y anchura) usando calibrador electrónico (Mitutoyo); se registró la ubicación de las lesiones aneurismáticas y la expresión morfológica de los 


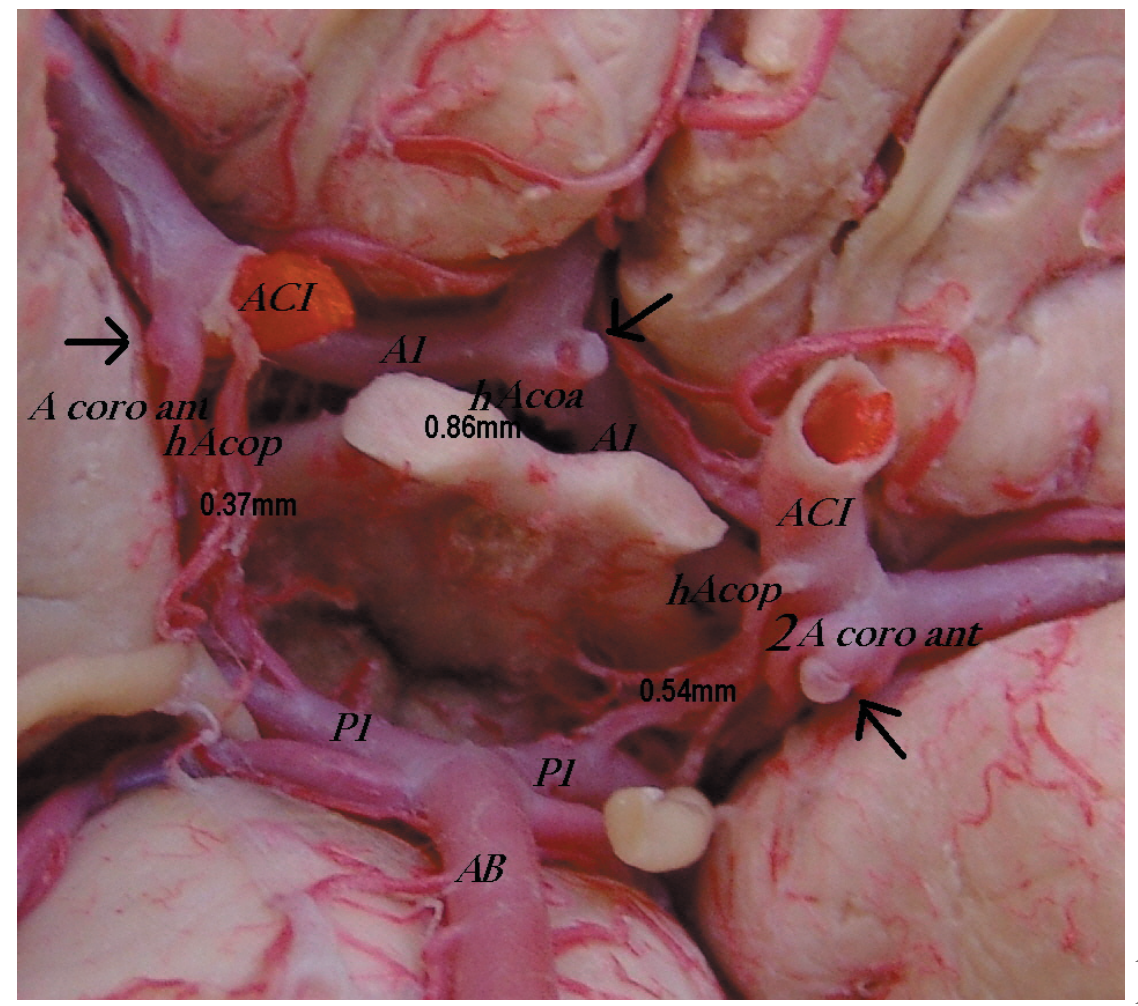

Figura 1. Triple lesión aneurismática: aneurismas de las arterias carótidas internas a nivel del origen de las arterias coroideas anteriores bilaterales $y$ aneurisma de la arteria comunicante anterior. hAcop: hipoplasia de la arteria comunicante posterior (calibres: derecha $0.37 \mathrm{~mm}$, izquierda $0.54 \mathrm{~mm})$. A coro ant: arteria coroidea anterior. 2 A coro ant: doble arteria coroidea anterior. A1: segmento precomunicante de la arteria cerebral anterior. hAcoa: hipoplasia arteria comunicante anterior. P1: segmento precomunicante de la arteria cerebral posterior. AB: arteria basilar.

\section{Tabla 1}

Distribución por grupos de edad y frecuencia de lesiones aneurismáticas en una muestra de población colombiana

\begin{tabular}{|c|c|c|c|c|}
\hline & \multicolumn{2}{|c|}{ Encéfalos } & \multicolumn{2}{c|}{ Aneurismas } \\
\hline Edad (años) & No. & $\%$ & No. & $\%$ \\
\hline $16-20$ & 7 & 5,6 & 0 & 0,0 \\
\hline $21-29$ & 32 & 25,6 & 0 & 0,0 \\
\hline $30-39$ & 44 & 35,2 & 1 & 16,7 \\
\hline $40-49$ & 25 & 20 & 1 & 16,7 \\
\hline $50-59$ & 11 & 8,8 & $2 *$ & 33,3 \\
\hline $60-65$ & 6 & 4,8 & $2 * *$ & 33,3 \\
\hline Total & $\mathbf{1 2 5}$ & $\mathbf{1 0 0}$ & $\mathbf{6}$ & $\mathbf{1 0 0}$ \\
\hline
\end{tabular}

No: Número; \%: Porcentaje. *Doble lesión aneurismática. **Triple lesión aneurismatica

segmentos de los polígonos asociados a estas lesiones; se consideró presencia de segmentos hipoplásicos cuando el calibre de éstos fue menor a $1 \mathrm{~mm}^{7,17}$ y asimetría si el diámetro de uno de los segmentos corresponde al $75 \%$ o menos del contralateral.

De cada una de las piezas evaluadas se obtuvieron imágenes fotográficas con cámara digital Sony DSC-F828. Los espécimenes evaluados se agruparon por grupos etáreos de la siguiente manera: $<20,20-29,30-39,40-9$ y $>60$ años; los registros obtenidos fueron incluidos en una base de datos y se analizaron comparativamente usando Microsoft Office Excel 2003.

\section{Resultados}

El promedio de edad de la muestra evaluada fue de 33,8 años DS 15,6; correspondiendo el mayor número de encéfalos al grupo de 30-39 años (35.2\%); se observaron nueve lesiones aneurismáticas en seis encéfalos (4.8\%); todos los aneurismas se presentaron en mayores de 30 años; Se presentaron aneurismas múltiples en dos $\mathrm{PW}$ (33.3\%), un encéfalo de un individuo entre 40-59 años presentó doble lesión y en otro del grupo mayor de 60 años se observo triple lesión aneurismática (Tabla 1), (Figura 1).

\section{Localización}

Ocho aneurismas $(88.8 \%)$ se ubicaron en el segmento anterior del polígono, siendo las estructuras más afectadas, el segmento supraclinoideo de la ACI con cuatro casos (44.4\%) y la AcoA, con tres casos (33.3\%). En el segmento posterior del polígono se encontró sólo un aneurisma (11.2\%) localizado en la bifurcación de la arteria basilar.

\section{Tamaño}

La media del tamaño de las lesiones aneurismáticas fue de $2.49 \mathrm{~mm}$ DS 0.37 IC 95\% 0.24; el aneurisma de mayor tamaño midió $3.15 \mathrm{~mm}$ y se ubicó lateral al origen de la 


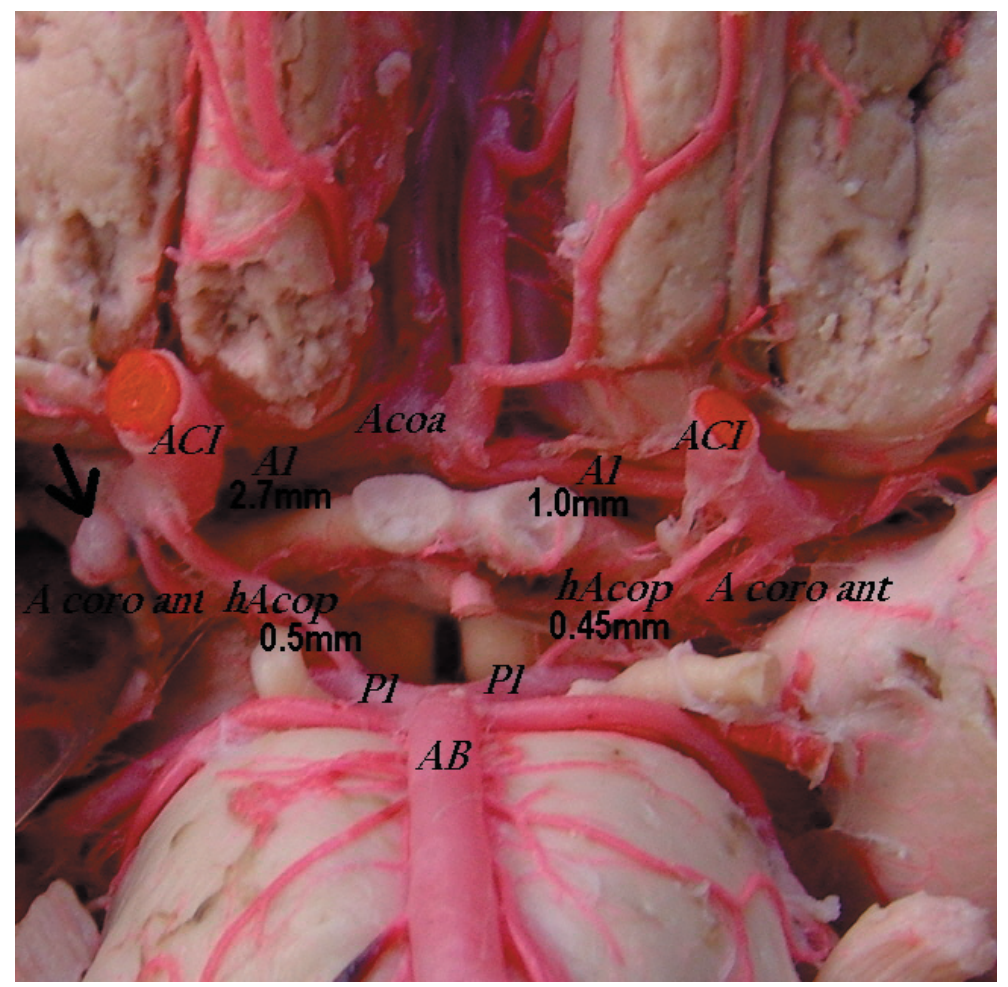

Figura 2. Aneurisma de la arteria carótida interna a nivel del origen de la arteria coroidea anterior: (lesión de mayor tamaño), asociada a hipoplasia bilateral de arteria comunicante posterior. hAcop: hipoplasia de la arteria comunicante posterior (calibres: derecha $0.5 \mathrm{~mm}$, izquierda $0.4 \mathrm{~mm})$. A coro ant: arteria coroidea anterior. A1: segmento precomunicante de la arteria cerebral anterior. P1: segmento precomunicante de la arteria cerebral posterior. AB: arteria basilar.

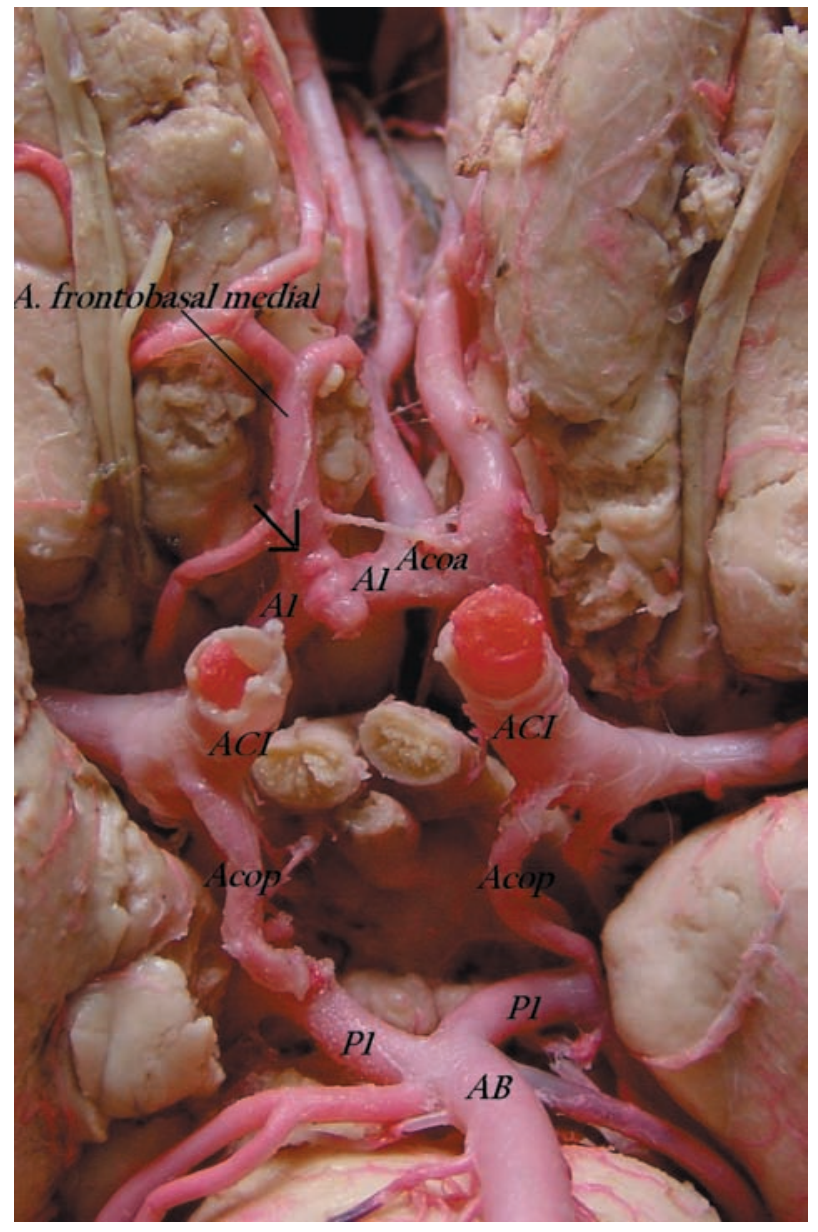

arteria coroidea anterior (Figura 2).

\section{Expresión morfológica asociada}

Todos los aneurismas se encontraron asociados a sitios de bifurcación arterial o de ramificaciones de las arterias primarias.

Aneurisma del segmento A1: la lesión aneurismática estuvo asociada a variación en el origen de la arteria frontobasal medial derecha, la cual se originó en el segmento A1 de la ACA, causando un giro adicional en su trayectoria (Figura 3).

Aneurismas de arteria carótida interna: se presentaron cuatro casos $(44,4 \%)$, tres de los cuales se localizaron a nivel del origen de la arteria coroidea anterior, estos se asociaron a hipoplasia bilateral de AcoP; uno de estos, se asoció además a duplicación de la arteria coroidea (Figura 1). El cuarto aneurisma de la ACI se presentó en el sitio de su bifurcación.

Aneurismas de arteria comunicante anterior: se encontraron tres casos (33.3\%), una de estas lesiones se asoció

Figura 3. Aneurisma de la arteria cerebral anterior (segmento precomunicante): asociada a variación en el origen de la arteria frontobasal medial. A1: segmento precomunicante de la arteria cerebral anterior. P1: segmento precomunicante de la arteria cerebral posterior. $A B$ : arteria basilar. 


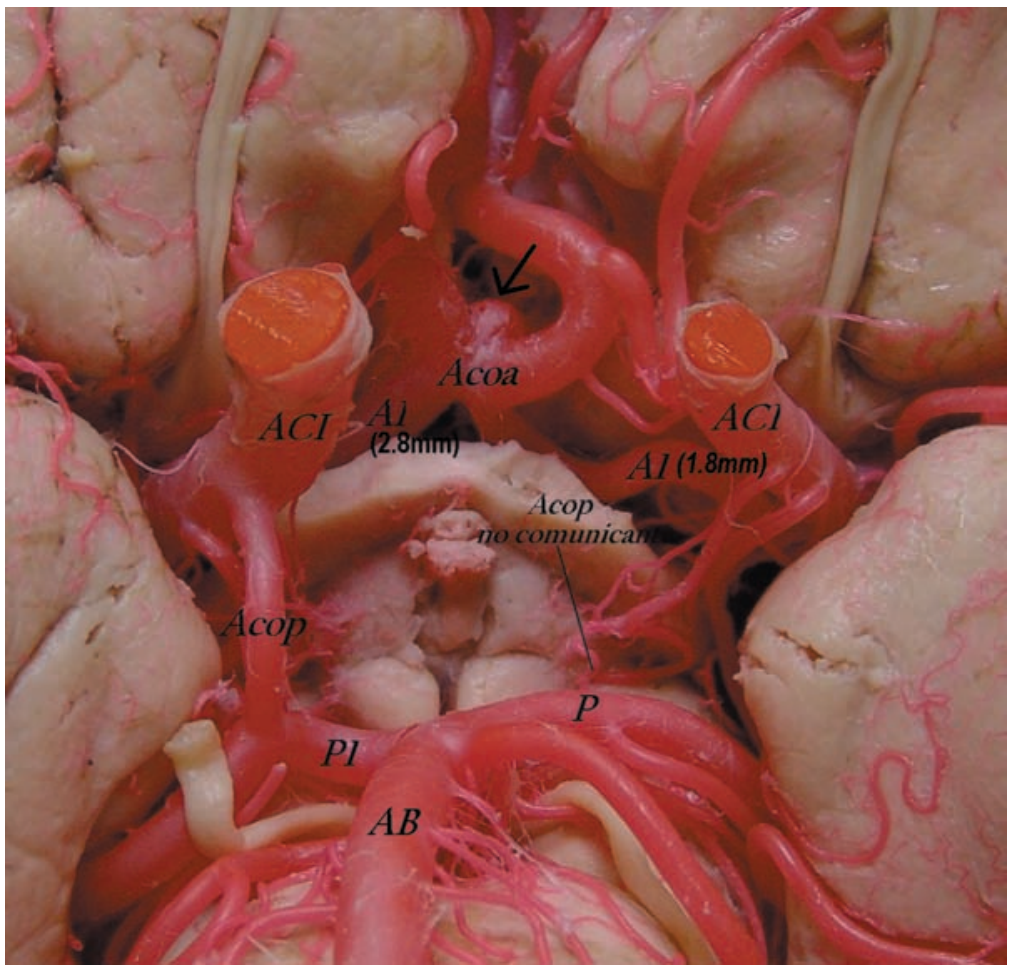

Figura 4. Aneurisma de la arteria comunicante anterior: asimetría de segmentos A1 (calibres: A1 derecha 2.8mm, Al izquierda 1.8mm), ausencia de la comunicante posterior izquierda. A1: segmento precomunicante de la arteria cerebral anterior. . Acop no comunicante: ausencia de comunicacion de la Acop con el sistema vertebrobasilar. A coro ant: arteria coroidea anterior. P1: segmento precomunicante de la arteria cerebral posterior. P: arteria cerebral posterior. AB: arteria basilar.

a hipoplasia de esta estructura (Figura 1), en una segunda muestra (Figura 4) se presentó asimetría de segmentos A1 $(2.84 / 1.84 \mathrm{~mm})$ y agenesia de la AcoP izquierda; en el tercer caso, se observó el aneurisma asociado a una fenestración de la arteria en forma de $\mathrm{H}$.

Todos los encéfalos con lesiones aneurismáticas presentaron alguna variación en la configuración del PW; dentro de éstas se destacaron asimetrías de la ACA en dos casos $(33.3 \%)$ y las variaciones de la AcoP (hipoplasia o agenesia), que se presentaron en el $83.3 \%$ de los encéfalos con aneurismas, con dos casos de hipoplasia unilateral, dos con bilateral $(33.3 \%)$ y un encéfalo con agenesia (16.7\%). Sólo uno de los polígonos tuvo diámetros normales para esta arteria. Se registró en el grupo de encéfalos sin aneurismas (119 encéfalos) la prevalencia de estas variaciones y se observó hipoplasia bilateral de AcoP en 32 muestras (26.9\%); hipoplasia unilateral en $26.9 \%$ (13 casos, $10.9 \%$ en el lado izquierdo y 19 casos $16 \%$, en el derecho), adicionalmente se encontraron cuatro casos de agenesia unilateral $(3,4 \%)$ y un caso $(0.8 \%)$ agenesia bilateral. En el $42.0 \%$ de los polígonos evaluados no se encontró variación de la AcoP.

\section{Discusión}

La frecuencia de aneurismas cerebrales no rotos en nuestro trabajo $(4,8 \%)$ es concordante con estudios previos $(0.2-9.9 \%)^{1,3,4,13,15,19,24}$. Esta entidad se ha descrito con mayor frecuencia en mujeres; sin embargo el estudio se realizó en encéfalos de individuos masculinos, por lo cual no tenemos resultados de frecuencia en la población femenina.

Al evaluar en nuestro estudio la prevalencia de aneurismas por grupos de edad, observamos la inexistencia de lesiones en menores de 30 años y el significativo incremento de éstas en los grupos mayores a 50 años ${ }^{13,15}$.

Adicionalmente se destaca la aparición de lesiones múltiples en estos grupos de mayor edad, con una frecuencia ligeramente superior a la señalada en la literatura ${ }^{3,13,15,25}$ (alrededor del 20\%). Estas observaciones podrían explicarse por la conjugación de variables de sustrato anatómico y los efectos deletéreos de factores de riesgo que se han involucrado en la génesis y progresión de estas lesiones, como hiperlipidemia, hipertensión, ateroesclerosis, tabaquismo, etc ${ }^{24,25}$.

La localización de los aneurismas no rotos del PW en el segmento anterior observados en este estudio $(88,8 \%)$ es similar a lo reportado en otros grupos poblacionales $(80 \%$ $92 \%$ ); el único caso que observamos en el segmento posterior del polígono $(11,2 \%)$, se presentó en la bifurcación basilar que corresponde a la ubicación mas frecuentemente reportada para este segmento ${ }^{3,4,6,18,28}$.

En nuestros hallazgos La ACI fue la estructura mas afectada con lesiones aneurismáticas $(44,4 \%)$, resultados que son concordantes con lo señalado por otros autores ${ }^{24,25,28}$. En contraste, otros estudios ${ }^{1,3,8,16}$ describen a la AcoA como el sitio más frecuente de asiento de aneurismas cerebrales; esta localización fue observada como la segunda mas fre- 
cuente (33.3\%) en nuestra serie.

En relación al nivel de origen de los aneurismas de la ACI, los estudios previos ${ }^{3,6,16,28}$ consideran al origen de la AcoP como el sitio mas común de localización de estas lesiones; se destaca que en la muestra examinada no se encontraron casos con esta localización, por el contrario, en tres de los cuatro espécimenes observados, la lesión aneurismática se localizó en el origen de la arteria coroidea anterior. Se hace relevante la coexistencia de esta lesión, con hipoplasia bilateral de la AcoP, variación anatómica que ha sido descrita con alta frecuencia por algunos auto$\operatorname{res}^{7,17}$.

Un estudio con material de autopsia en población masculina iraní ${ }^{7}$, mostró variaciones de la AcoP con frecuencias muy similares a las encontradas en nuestro trabajo, ya que informan presencia de hipoplasia derecha en $16 \%$, izquierda en $11 \%$ y bilateral en 33\%; adicionalmente señalan coexistencia con aneurismas de la AcoP en un caso; Un aspecto a resaltar es la inexistencia de aneurismas en la arteria coroidea anterior en este estudio a pesar de frecuencias similares de hipoplasias en la AcoP, asociación que fue evidente en nuestra muestra. La hipoplasia de ACP y la AcoP fue la variación más frecuentemente observada en un estudio en población Indú, pero este no evaluó la presencia de lesiones aneurismáticas ${ }^{17}$, En un estudio tomográfico realizado en una muestra de población colombiana, se estableció una frecuencia de $36.8 \%$ para hipoplasia bilateral y $21 \%$ hipoplasia unilateral (memorias tercer congreso Colombiano de morfología 2007) resultados que son concordantes con los hallazgos del presente estudio (58.0\%), igualmente este no evaluaron asociación con aneurismas. El alto número de aneurismas localizados en el origen de la arteria coroidea anterior evidenciado en nuestro estudio y su asociación a hipoplasia de la AcoP nos lleva a plantear la posibilidad de una relación causal entre éstas, sin embargo, por la alta frecuencia de esta variación en la población colombiana y en otros grupos poblacionales, sin evidencia de lesiones aneurismáticas asociadas, nos permite plantear que podría corresponder a un hallazgo coincidental.

En la muestra evaluada todos los encéfalos con lesiones aneurismáticas presentaron configuración asimétrica del circulo de Willis lo cual se ha correlacionado como factor etiopatogénico de aneurismas principalmente en el origen de la comunicante anterior ${ }^{1,5,11,31}$. En un estudio autópsico en población china se encontró asimetría de los segmentos A1, con una frecuencia de $13.30 \%$ y en un caso se asoció a lesión aneurismática de la arteria comunicante anterior $^{31}$; en la población española se encontró aneurismas en esta localización en el $71 \%$ de casos donde se estableció dominancia de flujo evaluada por estudio hemodinámico, mientras que en el grupo sin dominancia la frecuencia de aneurismas fue mucho menor $(3.9 \%)^{11}$; En nuestro estudio se observó un caso en el cual se presentó coexistencia de asimetría del segmento A1 derecho asociado a aneurisma de la AcoA, lo cual puede es un factor importante en el origen y desarrollo de estas lesiones.

Aproximadamente la mitad de los aneurismas se localizan en las bifurcaciones arteriales, siendo menos frecuentes los no relacionados con éstas; asociación que ha llevado a plantear que uno de los factores etiopatogénicos de las lesiones aneurismáticas es la impresión de la corriente de flujo en la pared de un vaso siendo ésta mayor en las bifurcaciones arteriales ${ }^{5,6,14,21-23}$. Los resultados de nuestro estudio apoyan este planteamiento ya que la totalidad de los casos se asociaron a ramificaciones o bifurcaciones de las arterias primarias.

A pesar que es aceptado que la ruptura de los aneurismas se asocia a su mayor tamaño y reconociendo que el diámetro mayor de las lesiones aneurismáticas registradas en este trabajo fue de tan sólo $3.15 \mathrm{~mm}$, su posible progresión y ruptura no debe relegarse de las causas de morbimortalidad en la población colombiana.

$\mathrm{La}$ inexistencia de trabajos morfológicos sobre aneurismas no rotos en este grupo poblacional, permiten que estos resultados sean tomados como referentes para futuros estudios.

\section{Bibliografía}

1. Aydin, I.H., Takci, E., Kadioglu, H.H., et al.: Vascular Variations Associated with Anterior Communicating Artery Aneurysm - An Intraoperative Study. Minim. Invas. Neurosurg. 1997; 40: 17-21.

2. Carter, B.S., Sheth, S., Chang, E., et al.: Epidemiology of the size distribution of intracranial bifurcation aneurysms: smaller size of distal aneurysms and increasing size of unruptured aneurysms with age. Neurosurgery 2006; 58: 217- 223.

3. Castel, J.P.: Los Aneurismas Intracraneanos. Decq P. Keravel Y. Velasco F. Neurogirugia. 1 ed. Mexico 1999: 477513.

4. Chairman., Rosai, J.: Surgical Pathology. Department of pathology National Cancer Institute. Milan, Italia 2000; pp 2471- 2473.

5. Charbel, F.T., Seyfried, D., Mehta, B., et al.: Dominant A1: Angiographic and clinical correlations with anterior cummunicating artery aneurysms. Neurological Research 1991; 13: 253-256.

6. De la Monte, S., Moore, W.G., Hutchins, G.M.: Risk Factors for the Development and Rupture of Intracranial Berry Aneurysms. The American Journal of Medicine 1985; 78: 957-964.

7. Eftekhar, B., Dadmehr, M., Ansari, S., et al.: Are the distributions of variations of circle of Willis different in different populations? - Results of an anatomical study and review of literature. BMC Neurology 2006; 6: 1-9. 
8. Forget, T.R., Benitez, R., Veznedaroglu, E., et al.: A Review of Size and Location of Ruptured Intracranial Aneurysms. Neurosurgery 2001; 49: 1322- 1326.

9. Gewirtz, R.J., Awad, I.A.: Giant Aneurysms of the Proximal Anterior Cerebral Artery: Report of Three Cases. Neurosurgery 1993; 33: 120-124.

10. Gijn, J.V., Kerr R.S., Rinkel G.J.E.: Subarachnoid haemorrhage. The Lancet 2007; 369: 306-318.

11. González- Llanos, F.. Pascual, J.. Roda, J.: Bases anatómicas y hemodinámicas del complejo de la arteria comunicante anterior. Neurocirugía 2002; 4: 285-298.

12. Hashimoto, M., Urasaki, E., Tsujigami S., Yokota, A.: Ruptured Aneurysm Associated With Partially Duplicated Posterior Communicating Artery. Neurol Med Chir 2002; 42 : 23-26.

13. Inagawa T. Hirano A.: Autopsy study of unruptured incidental intracranial aneurysm. Surg Neurol. 1990; 34: 361365.

14. Ingebrigtsen, T., Morgan, M.K., Faulder, K., et al.: Bifurcation geometry and presence of cerebral artery aneurysms. J. Neurosurg. 2004; 101: 108-113.

15. Iwamoto, H., Kiyohara, Y., Fujishima, M., et al.: Prevalence of Intracranial Saccular Aneurysms in a Japanese Community Based on a Consecutive Autopsy Series During a 30-Year Observation Period. The Hisayama Study. Stroke. 1999; 30: 1390-1395.

16. Kalula, N., Kayembe, K.N., Sasahara, M., et al.: Cerebral aneurysms and variation in the circle of Willis. Stroke 1984; 15: 846-850.

17. Kamath S.: Observations on the length and diameter of vessels forming the circle of Willis. J. Anat. 1981; 133: 419423

18. Martinez, F., Spagnuolo, E., Calvo- Rubal, A.: Variaciones del sector anterior del Polígono de Willis. Correlación anatomoangiográfica y su implicancia en la cirugía de aneurismas intracraneanos. Neurocirugia 2004; 15: 578-589.

19. Massachusetts Medical Society.: Unruptured intracranial aneurysms - risk of rupture and risks of surgical intervention. N Engl J Med 1998; 339: 1725-1733.

20. Mc Cormick, W.F., Acosta-Rua, G.J.: The size of intracranial saccular aneurysms. An autopsy Study. J Neurosurg 1970; 33: 422-427.

21. Meng, H., Wang, Z., Hoi, Y., et al.: Complex Hemodynamics at the Apex of an Arterial Bifurcation Induces Vascular
Remodeling Resembling Cerebral Aneurysm Initiation. Stroke 2007; 38: 1924-1931.

22. Mérei, F.T., Gallyas,F.: Intracranial Saccular Aneurysm Unrelated to Bifurcation. Acta Neurochirurgica 1981; 57: 247-256.

23. Milet, L.E.: Historia natural de los aneurismas intracraneales. Aneurismas sin ruptura. Rev Argent Neuroc 2006; 20: 19- 24.

24. Minyard, A.: Parker J. Intracranial Saccular (berry) Aneurysm: A brief overview. Southern Medical Journal. 1997; 90:672-677

25. Miyazawa, N., Akiyama, I., Yamagata, Z.: Risk factors for growth of unruptured intracranial aneurysms: follow-up study by serial 0.5 -t magnetic resonance angiography. Neurosurgery 2006; 58: 1047-1053.

26. Nystrom, S.H.M.: On Factors Related to Growth and Rupture of Intracranial Aneurysms. Acta Neuropath 1970; 16: 64-72.

27. Pradilla, G., Vesga, B.E., León-Sarmiento, F.E., grupo GENECO.: Estudio neuroepidemiológico nacional (EPINEURO) colombiano. Rev Panam Salud Public 2003; 14: 104- 111.

28. Rhoton, A.L.: Aneurysms. Neurosurgery 2002; 51: 121-158.

29. Silva, F.A., Zarruk, J.G., Quintero, C., et al.: Enfermedad cerebrovascular en Colombia. Rev. Colomb. Cardiol. 2006; 13: 85-89.

30. Takeuchi, Y., Guevara, J.G.: Prevalencia de las enfermedades neurológicas en el Valle del Cauca. Estudio Neuroepidemiológico Nacional. Colombia Med 1999; 30: 74-81.

31. Tao, X., Jiang Yu, X., Bhattarai, B., et al.: Microsurgical anatomy of the anterior communicating artery complex in adult Chinese heads. Surgical Neurology. 2006; 65: 155-16.

Quintero-Oliveros, S.T.; Ballesteros-Acuña, L.E.; AyalaPimentel, J.O.; Forero-Porras, P.L.: Características morfológicas de aneurimas cerebrales del polígono de Willis: estudio anatómico directo. Nreurocirugía 2009; 20: 110-116.

Correspondencia postal: Silvia Tatiana Quintero Oliveros MD. Carrera 32 Numero 31-29 Facultad de Salud. Departamento de Ciencias Basicas. Universidad Industrial de Santander. Bucaramanga. Colombia. 